\title{
Beam Test of The SDC Barrel HM Calorimeter Test Module
}

L. Balka,V. Guarino,N. Hill,D. Jankowski,C. Keyser,T. Kirk, L. Kocenko,D. Morgan,J. Nasiatka,E. Petereit,L. Price,J. Proudfoot,H. Spinka, D. Underwood,R. Wagner,K. Wood High Energy Physics Division Argonne National Laboratory, Argonne Illinois 60499

\begin{abstract}
The SDC barrel electromagnetice calorimeter test module was exposed to beams of high energy pions and electrons in the MP9 test beam at Fermilab in the fall of 1991. Data were collected on resolution, light yield, signal timing and hermiticity. These data demonstrated that the design met the specifications for the barrel electromagnetic calorimeter of the Solenoidal Detector Collaboration (SDC).
\end{abstract}

\section{Introduction}

The SDC ${ }^{1,2}$ chose to build a sampling calorimeter using scintillator plate with wavelength-shifting fiber readout as the sampling medium, and lead and iron plates for the absorber media in the electromagnetic and hadronic calorimeters respectively. The overall structure is formed by assembling submodules, each of which covers a fraction of the azimuthal solid angle (wedges). The electromagnetic calorimeter wedges are fabricated by casting lead sheets within a low mass stainless steel frame. Aluminum spacer plates inserted in the casting provide for the slots into which scintillator tiles may be inserted. To evaluate the expected performance of this design, two such wedges which were $1 / 6$ of the length of the full scale device in the $z$ direction but covered the full azimuthal angle were cast and instrumented. The $\mathrm{z}$ segmentation for this test module comprised 5 towers along its length and 2 in azimuth each of approximately $10 \times 10 \mathrm{~cm}$ in area. These wedges were mounted on a remotely moveable table and exposed to high energy beams in the Fermilab MP9 area. ${ }^{3}$ The gap between the two wedges was arranged to be comparable to that which would be realised in the full calorimeter. A schematic of the layout is shown in Fig. 1. The key items under study were the energy resolution of the device and its hermiticity. However, several other aspects of the design were investigated and are reported below.

\section{Test Beam Instrumentation}

The MP9 area at Fermilab was well known to several members of the group embarking on these test module measurements as it was used previously for a polarised beam experiment in which they were participants. 4 The beam line instru-

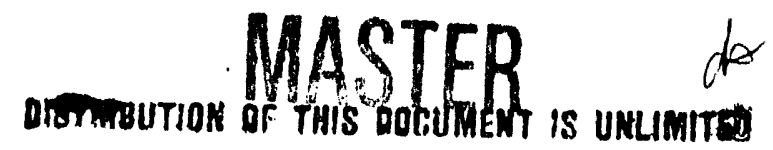


SDC TILE/FIBER CALORIMETER

Truncated EMC Module for Testbeam

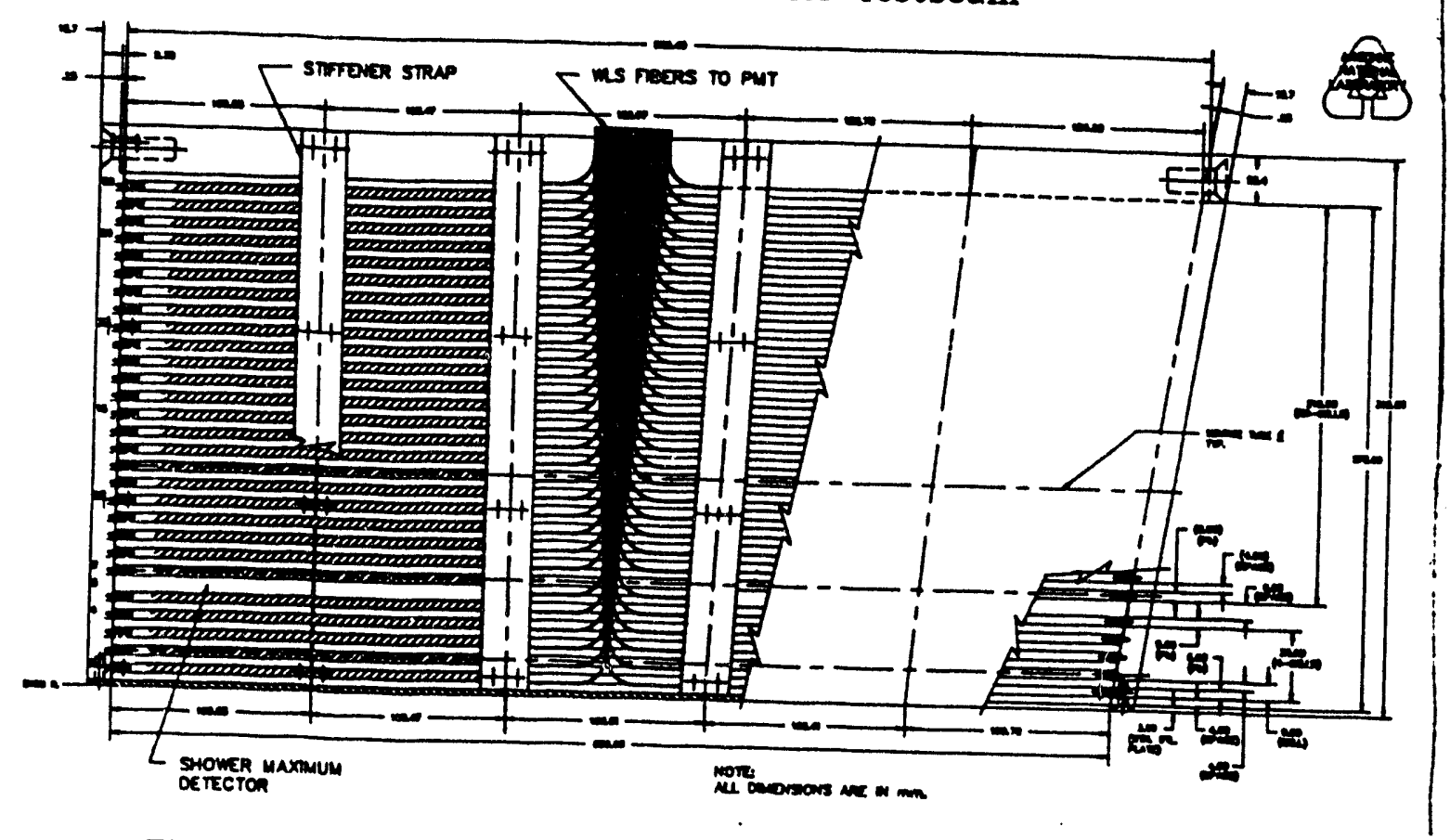

Figure 1: The barrel electromagnetic calorimeter test module (schematic).

mentation comprised multiwire proportional chambers, scintillator hodoscopes and a dipole magnet for momentum tagging. Below $40 \mathrm{GeV}$, the momentum resolution of the beamline is primarily limited by multiple scattering off material in the beamline and is shown in Fig. 2. Particle identification was available using two threshold Cerenkov counters. The electron contamination in the pion beam was typically a few percent. The calorimeter towers were read out using R580-17 photomultiplier tubes coupled to a LRS2249W charge integrating ADC. The data were readout via CAMAC using an online system based on VaxOnline. Realtime monitoring was
available via PAW.

\section{Electromagnetic Calorimeter Performance.}

\section{s.1. Resolution}

The resolution of the electromagnetic calorimeter has been a topic of rather heated debate within the SDC. There is necessarily a desire within the collaboration to build the best possible device. However, this desire had to be tempered with the fiscal contraint of building an economically feasible detector. This lead to the specification that the stochastic term in the energy resolution be less than $15 \%$ and the constant term less than $1 \%$. These specifications then imply that the lead sampling be $4 \mathrm{~mm}$, that the lead plate thickness uniformity be better than of order $2 \%$ (from EGS studies) and the light yield be better than 2 photo-electrons/MIP/scintillator lajer. The plate thickness and light yield for the components used in the first of the two test modules is shown in Fig. 3. It should be noted that when built the SDC specifications had not been defined and therefore $5 \mathrm{~mm}$ lead plate sampling was used. However, in all other aspects, this device met the eventual specifications for the components and there was no obvious limitation arising from the modest 
Monte Carlo Momentum Spread of MP Beam

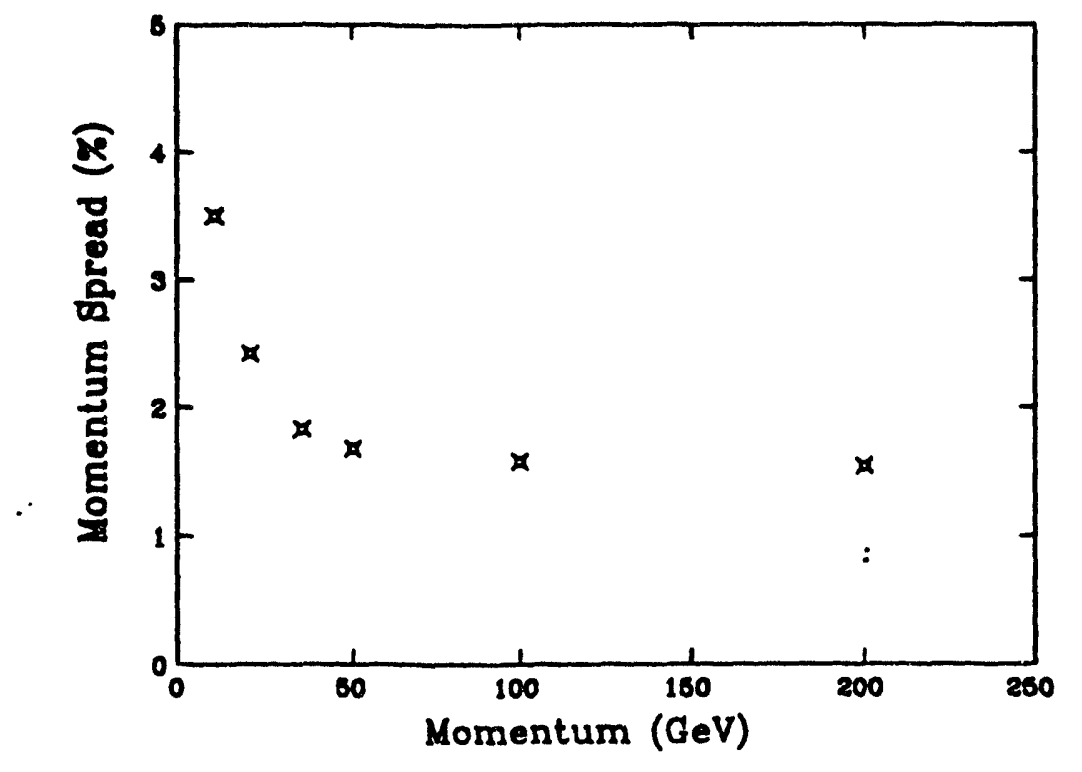

Figure 2: A Monte Carlo calculation of the multiple scattering limit on the momentum determination from material in the bean tagging system.

difference in plate thickness.

The resolution was one of the first performance characteristics studied in the test beam using high energy electrons. Figure 4 shows the measured resolution as a function of energy for electrons impinging on the center of the second tower in $z$ in the module (chosen to ensure good containment). In addition, to verify the photo-statistics, data were taken with a 2.6 times attentuating neutral density filter placed between the fibers reading the phototube and the photocathode. This gave an answer of 2 pe's per layer.

Following this result, further studies were made using the bench setup to study the discrepancy between the expected photo-electron yield of about 3 pe'smip and that measured in the beam of approximately 2 pe'smip. This was traced to the fact that the quantum efficiency of the tubes being used was dependent on the operating voltage. By an unfortunate turn of events we had chosen to operate in the test beam at lower voltage in order to maximise the dynamic range in beam energy when restricted to the 11 bit readout of the ADC. This resulted in an loss of approximately $30 \%$ in photo-cathode quantum efficiency (Fig. 5.)

\subsection{Hermeticity and Uniformity}

The tower stucture of this calorimeter is formed by the boundaries between tiles, which allow no scintillation light to pass. Several classes of such boundaries exist in the SDC design. The full calorimeter is assembled by connecting together 

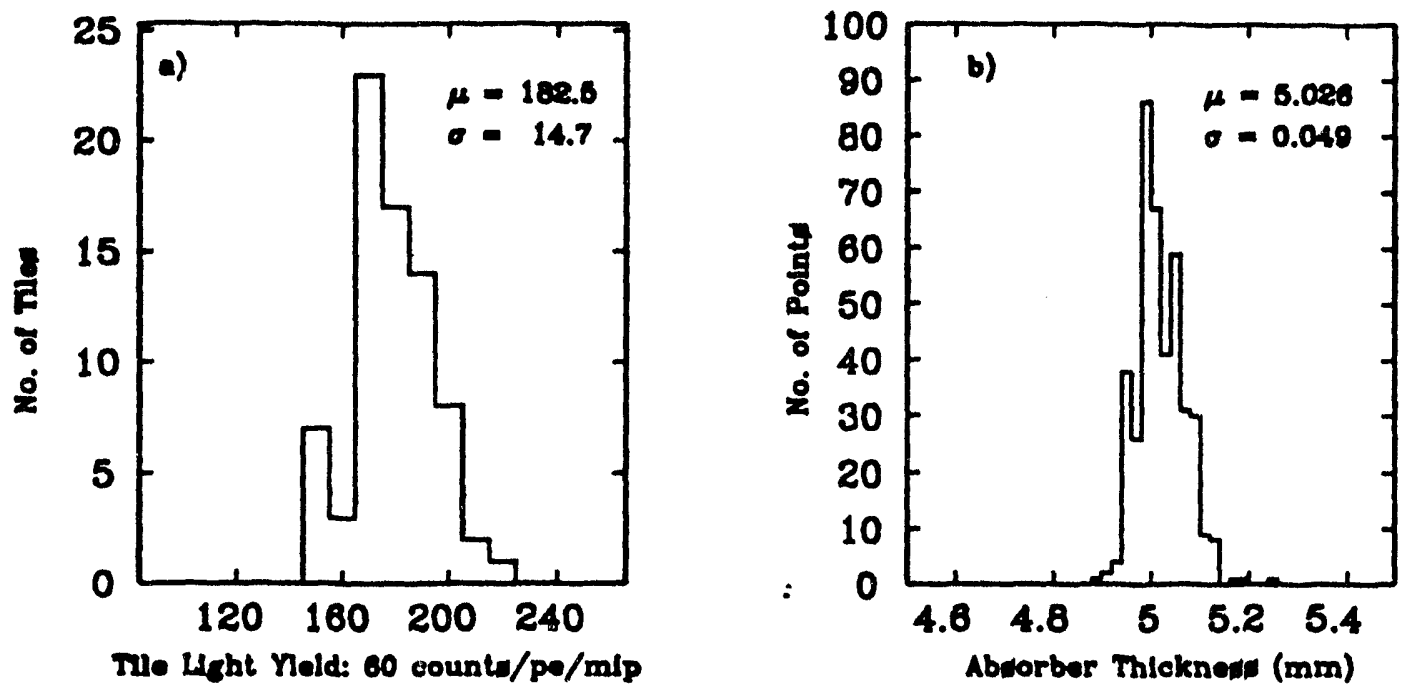

Figure 3: Uniformity of component elements of the first test module constructed: a) scintillator tile light yield measured using $3 \mathrm{MeV}$ electrons from a Ruthinuium source; b) lead plate thickness measured for three points per plate.

Tile/Fiber EMC Resolution

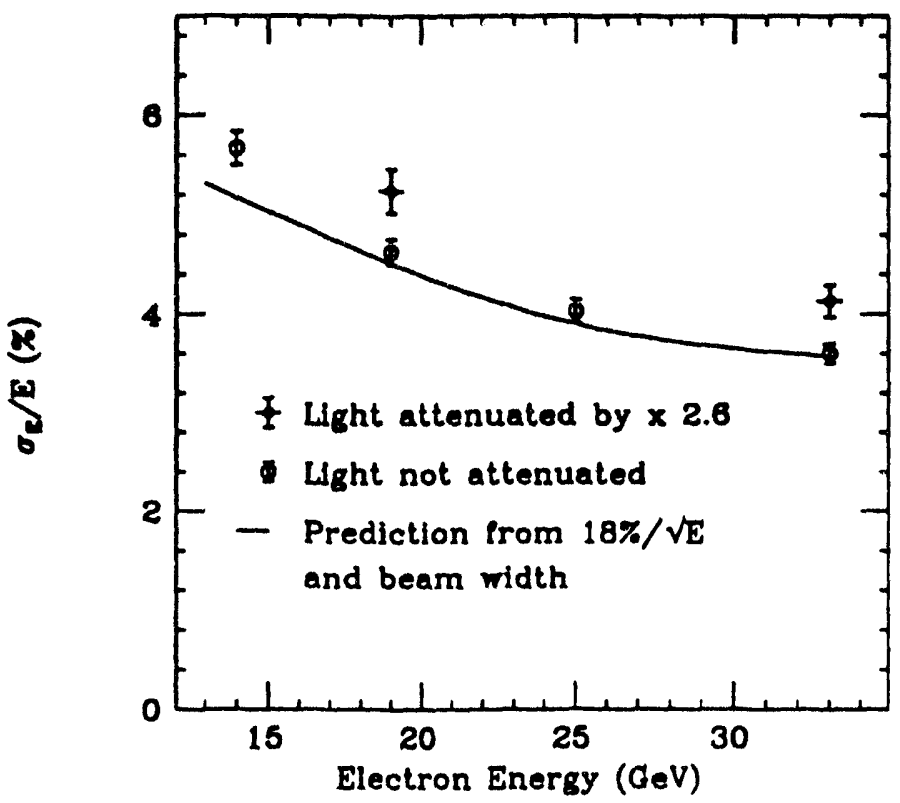

Figure 4: C'alorimeter energy resolution for electrons with energies betwen l0) and :0) (irl: The solid line is an ECS prediction in which t $f_{1}$. effects of photo-statistics. sampling fluctuations and momentum tagging resolution have been included. 


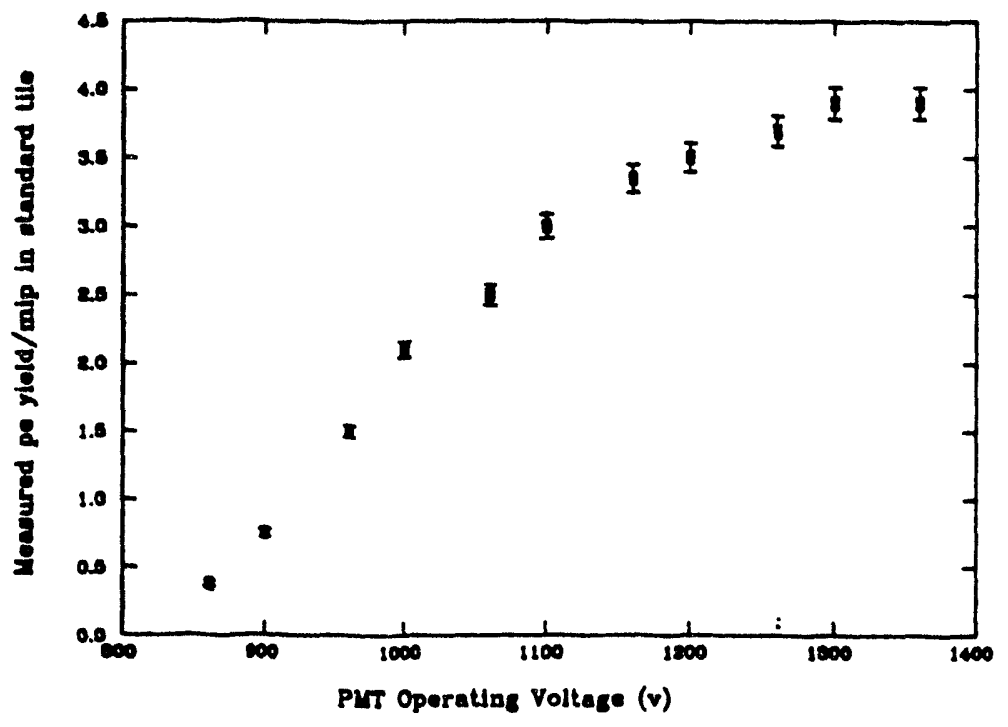

Figure 5: R580 photo-electron yield as a function of operating voltage

wedges, each of which cover 0.2 radians in azimuth. The crack between these wedges is used to route the readout fiber to the photon detectors. This fiber routing gap is $4.5 \mathrm{~mm}$ total and contains two sheets of fibers, thin stainless steel skins and a physical gap to prevent azimuthal load transfers within the stucture. As the wedge subassembly is $44 \mathrm{~cm}$ in azimuth this gap contribute of order $1 \%$ loss of acceptance (though it contributes a somewhat larger loss of good fiducial volume). A second azimuthal boundary is the result of the tower structure of the calorimeter. Each wedge has 4 towers in azimuth (covering a $\delta \phi$ of 0.05 radians) formed by individual tiles separated by spaces of approximately $0.3 \mathrm{~mm}$. EGS studies of the effect of such gaps in the sampling medium show that they will contribute less then $0.1 \%$ to the constant term in the resolution. Finally, the casting technology used to fabricate the absorber structure requires $0.5 \mathrm{~mm}$ stainless steel bulkheads at intervals of $0.1 \eta$ to support the lead plates (except in the slot containing the shower maximum detector where this spacing is increased to $0.2 \mathrm{\eta})$. In addition, to the loss of sampling medium, these bulkheads also absorb part of the shower energy and were the subject of considerable study. GEANT studies indicated that all of the apparent loss of resolution could be recovered by simply correcting the observed signal as a function of position. This conclusion was confirmed in testbeam measurements of a prototype electromagnetic calorimeter test module. Figure 5 shows the uncorrected signal as a function of impact position in $z$ along the face of the calorimeter. The resolution as a function of position, for the response corrected by the parameterisation, is shown in Fig. 6, where there the bulkheads are no longer visible. The fraction of energy lost in the cracks between tiles in a single wedge was studied in order to determine a specification for their placement. These data are shown in Fig. 7, where they are compared to a GEANT calculation and a numerical estimate based on the CDF electromagnetic shower parameteristion. The agreement is good, and the conclusion 


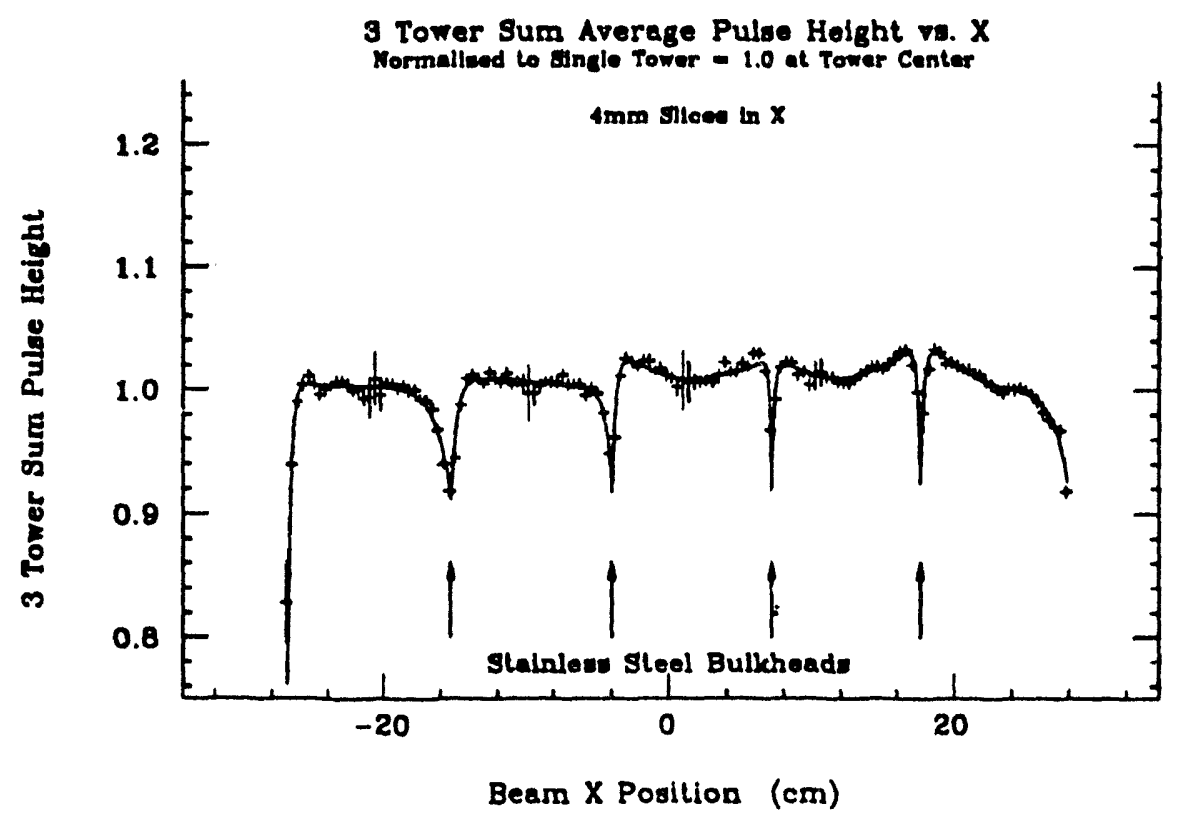

Figure 6: Cast lead electromagnetic calorimeter prototype : the uncorrected response to $35 \mathrm{GeV}$ electrons as a function of impact point. The curve is a fit to the data. The arrows indicate the position of bulkheads.

made was that the placement of tiles should provide for a maximum gap between scintillators of less than $0.4 \mathrm{~mm}$.

\subsection{Linearity}

For part of the running time it was possible to use very high energy beams of electrons. The rate at the highest energy was low due to a flux limitation and therefore it was only possible to establish the linearity of the device (some non-linearity is expected due to back leakage). The response of the calorimeter to electrons from 35 to $150 \mathrm{GeV}$ is shown in Fig. 8. The observed linearity is qualitatively good. A more quantitative statement proved impossible to due magnetic field saturation in the momentum analysing magnet (which was also part of the beam transport), which limited the precision with which the absolute momentum scale was known at high energy.

\subsection{Readout Speed}

It has long been recognised that scintillating calorimetry offers low noise signals with fast readout speed. This was one of the main advantages of this technology which lead the SDC to adopt it over its main contendor, a liquid argon calorimeter. There was however concern within the collaboration that this high speed readout could not be realised in practice, given the reflecting cavity formed by the wrapping 
Corr. 3 Tower Sum Pulge Height Resolution va. $X$

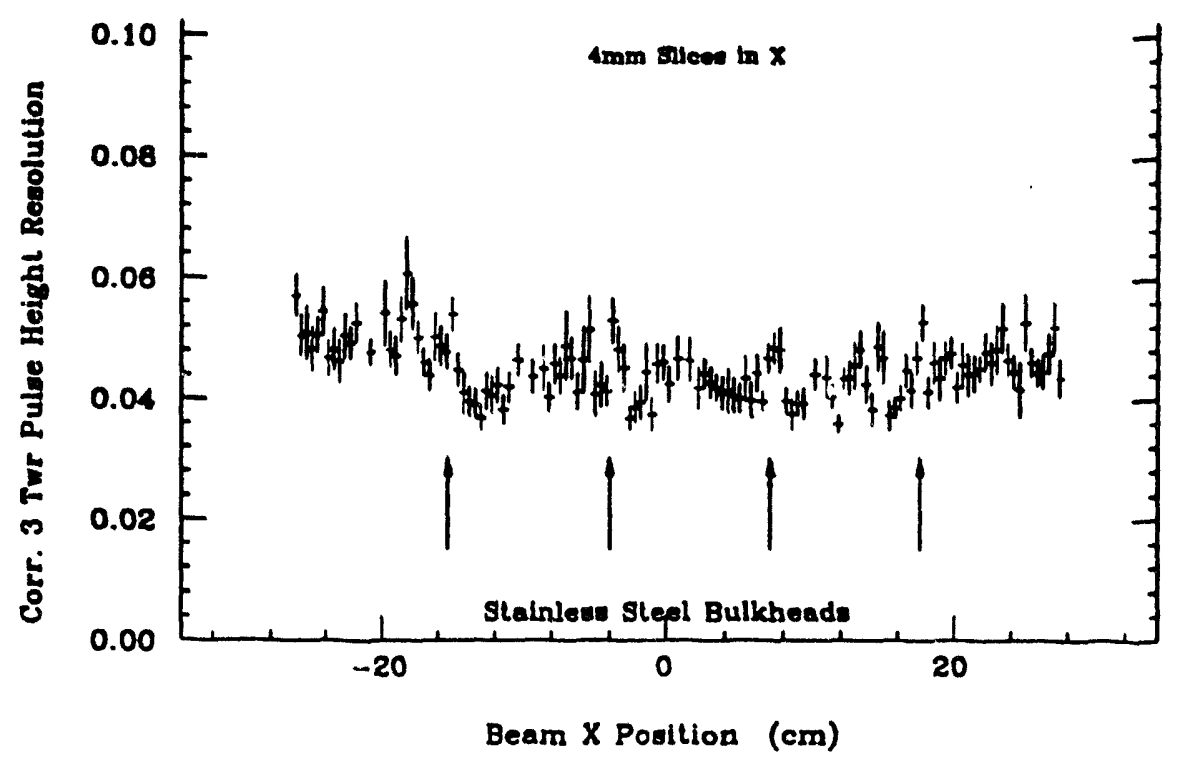

Figure i: Cast lead electromagnetic calorimeter prototype: The energy resolution for $35 \mathrm{GeV}$ electrons, using pulseteight corrected for loss of signal response in the bulkhead regions.

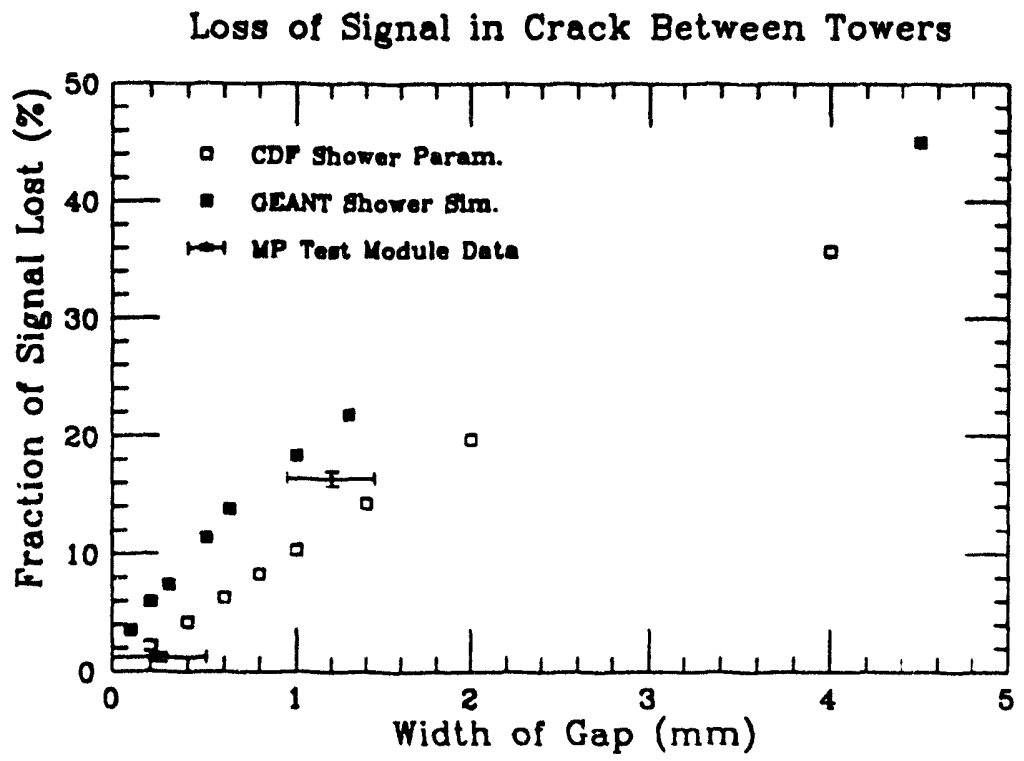

Figure 8: Loss of signal resulting from gaps between scintillator tiles within a single wedge. 


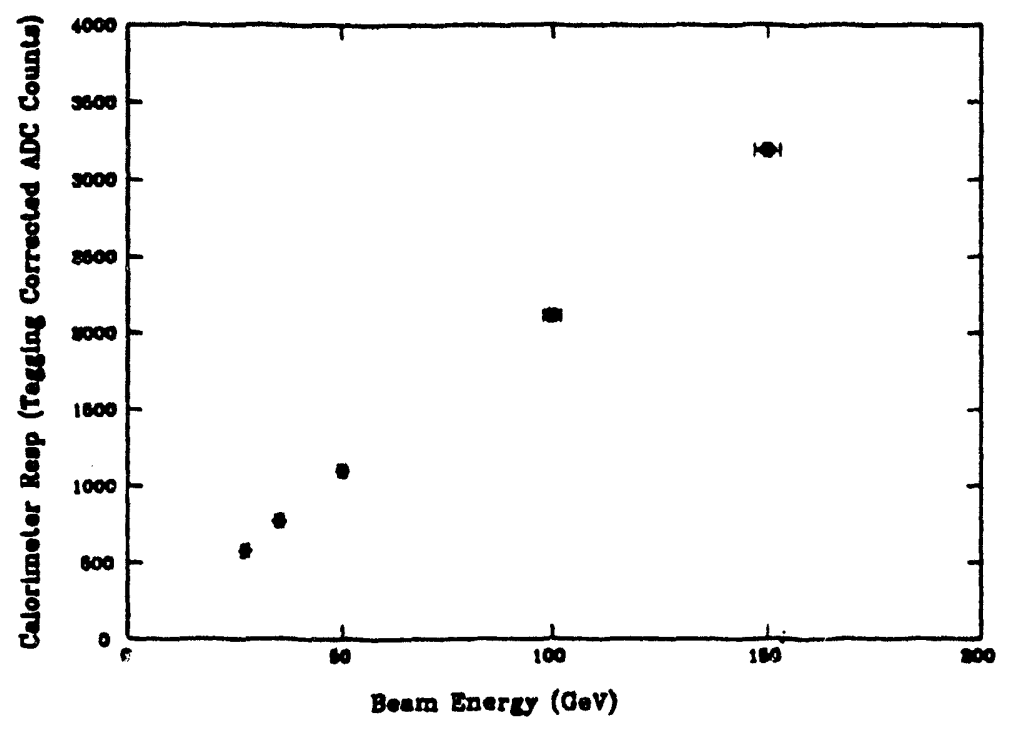

Figure 9: Calorimeter response to electrons as a function of energy

on the scintillator tiles. Following completion of the the basic energy measurements, a study of the energy response and resolution as a function of integration gate was carried out. This is shown in Fig. 9, which shows that a gate length of about 40 nsec is required to allow maximimum response and resolution.

\section{Performance Summary}

The SDC have chosen a conceptually rather simple technology for the central calorimeter. This uses the concept of embedded wavelength shifting fibers to couple scintillation light from tiles in a sampling calorimeter to a photon detector, with a minimum of uninstrumented detector. In practice however, this approach has opened some significant technological issues: mechanical support of a large structure with tight tolerances and small gaps; optical uniformity in a large number of scintillator tiles and many kilometers of wavelength shifting and plastic optical fiber. We have constructed and tested in high energy beams two test modules following this design concept. These have performed as expected and met the general perfromance specifications of the SDC.

\section{Acknowledgements}

Many individuals were involved in the setup and operation of the MP9 beamline for these tests, other than those whose interest was the electromagnetic calorimeter. Their interest lay in testing shower maximum detectors with novel readouts or the cesium calibration system. However, the test was run as a combineded effort and without their support this work could not have been accomplished. In particular, we wish to thank Angel Wang, Prisca Cushman, Anwar 

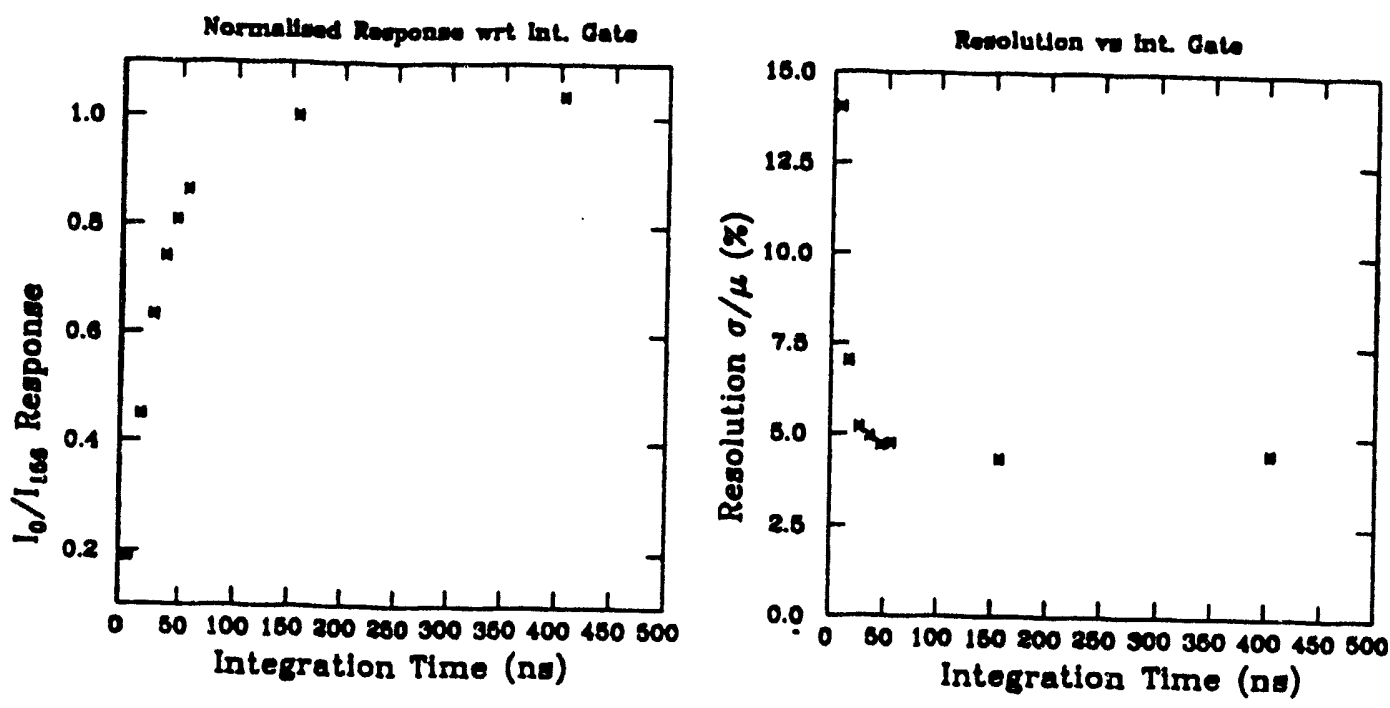

Figure 10: Energy resolution and response to $35 \mathrm{GeV}$ electrons as a function of signal integration gate.

Batti and Suen Hou for their work in developing and running the data acquisition system. The source calibration system was provided by Virgil Barnes and the photomultiplier HV control system by Luke Mo and Bin Lu. We are indebted to Robert Stanek, David Lopiano, and David Grosnick, ANL collaborators on E-704, and Carlos Hojvat for their assistance in setting up the veamline intrumentation. We also express our gratitude to Philip Melese, who assisted us in the instrumentation of the first test module and two exceptional students from Northeastern University, Doug Ruuska and Matt Hulbert, who contributed wherever needed. Finally, we acknowledge the support of the many members in the SDC calorimeter group who contributed in some way to this effort. This work supported by the US Department of Energy, Division of High Energy Physics, Contract W-31-109-ENG-38.

\section{References}

1. Letter of Intent by the Solenoidal Detector Collaboration, SDC-90-151 (1990).

2. The Solenoidal Detector Collaboration, Technical Design Report, SDC-92-201 (1992).

3. Proposal for Beam Test of Scintillator Calorimeter Prototypes, at Fermilab during FY 1991, E.I. Rosenberg et al., T-841 (1990).

4. The Design and Performance of the FNAL High-Energy Polarized Beam Facility, D.P. Grosnick et al., NIM A290 (1990) 269. 


\section{DISCLAIMER}

This report was prepared as an account of work sponsored by an agency of the United States Government. Neither the United States Government nor any agency thereof, nor any of their employees, makes any warranty, express or implied, or assumes any legal liability or responsibility for the accuracy, completeness, or usefulness of any information, apparatus, product, or process disclosed, or represents that its use would not infringe privately owned rights. Reference herein to any specific commercial product, process, or service by trade name, trademark, manufacturer, or otherwise does not necessarily constitute or imply its endorsement, recommendation, or favoring by the United States Government or any agency thereof. The views and opinions of authors expressed herein do not necessarily state or reflect those of the United States Government or any agency thereof. 

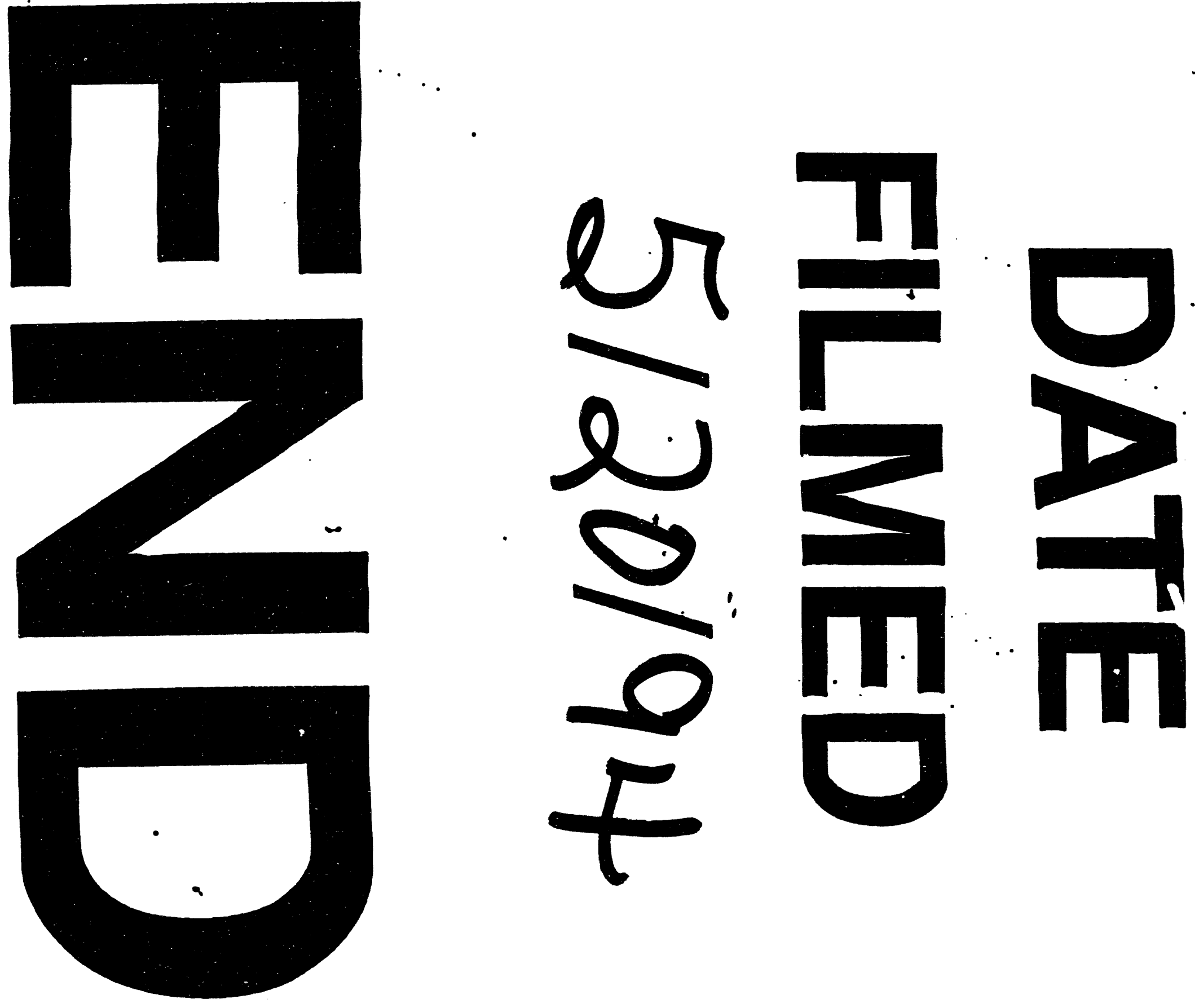
